\title{
Cuantificación del potencial energético undimotriz en las costas del Caribe Colombiano
}

\section{Quantification of the potential of wave energy in the Colombian Caribbean Coast}

\author{
Jaime Pérez Zapata \\ Universidad ITSA Soledad, Atlántico- \\ Colombia \\ Autor de correspondencia \\ Correo electrónico: \\ jperezz@itsa.edu.co
}

Wilson Becerra Colpas

Universidad ITSA Soledad,

Atlántico - Colombia

Información del artículo: recibido: 28 de Marzo 2017, aceptado: 09 de Agosto de 2017 https://10.17081/invinno.5.2.2758 


\section{Resumen}

En este documento se describen los avances en la cuantificación de la energía de las olas de la costa caribeña colombiana. La metodología utilizada servirá como punto de partida para establecer el potencial energético en Colombia para este tipo de suministro energético. En la actualidad, el potencial energético teórico estimado en las ondas de la costa cercana en la costa atlántica es de aproximadamente 1107 MW y esto fue posible con datos que se han recogido y procesado desde enero de 2014. Las ecuaciones que tienen en cuenta variables tales como: la densidad del agua de mar, la temperatura y la velocidad del viento en la zona. También muestra el progreso del diseño del sistema Near Shore para la verificación del potencial de energía de las olas que consiste en un dispositivo que transforma la energía cinética de las ondas del mar en energía potencial gravitacional que se va a usar para mover un generador de corriente alterna.

\section{Resumen}

This document describes the progress made regarding the quantification of colombian Caribbean shore potential wave energy. The used methodology will serve as a starting point to establish the energetic potential in Colombia in this energy way. Currently, The theoretical energetic potential estimated at the Near Shore in the Atlantic coast waves is approximately $1107 \mathrm{MW}$ and this was possible with data that has been collected and processed since January 2014. Equations that take into account variables such as: significant wave height, period, sea water density, temperature and wind speed in the area, has been applied. Also shows the progress of the Near Shore system design for the verification of the wave energetic potential consisting of a device which transforms kinetic energy of the sea waves into gravitational potential energy that is going to be used to move an alternating current generator.

\section{Palabras \\ Claves:}

Energía de ola, Altitud significativa de oleaje, Periodo de oleaje, Potencial de oleaje, Boya oceanográfica.

\section{Keywords}

Wave energy, Significant wave height, wave period, Wave power potential, Oceanographic buoy. 


\section{Introducción}

Este documento describe los avances realizados en la cuantificación del potencial de energía undimotriz (aquella que se obtiene mediante las olas del mar) [1] en las costas del Caribe colombiano. Hasta el momento, este potencial solo se ha cuantificado en el departamento del Atlántico. Pero, justamente, la metodología utilizada servirá como punto de partida para establecer el potencial del Caribe colombiano en este tipo de Fuente no Convencional de Energía Renovable (FNCER) y con esto atraer el interés de la comunidad investigadora nacional e internacional en la implementación de tecnologías que aprovechen este tipo de energía primaria para convertirla en energía eléctrica. Así, se logrará una importante transferencia de conocimientos y detecnología.

Se empieza por admitir que en Colombia, la energía undimotriz es poco conocida y utilizada. Por ello, en términos generales, se ignora el gran potencial energético que poseen las olas, a lo que se debe sumar la gran extensión de costas que posee el país. Para cumplir con el objetivo de este trabajo, se requería conocer, por tanto, este potencial energético y esto fue posible con la aplicación de ecuaciones consultadas, las cuales tienen en cuenta variables como: altura de la ola, periodo de las olas, densidad del agua de mar, temperatura y velocidad del viento en la zona. Estos datos han sido recopilados en conjunto con la Dirección General Marítima (DIMAR) y el Centro de Investigaciones Oceanográficas $(\mathrm{CIOH})$, adscritos al Ministerio de Defensa Nacional (MINDEFENSA) [2]. Además, han sido procesados desde el mes de enero de 2014, es decir, que suman un historial de datos mayor a tres años. Si bien es cierto que aun así se requiere un mayor historial de datos, consideramos que los datos procesados dan confiabilidad a los cálculos realizados para la determinación del potencial energético undimotriz de la zona objetivo, dado que el comportamiento de las olas en el área de estudio muestra poca variabilidad de un año a otro. No se realizan comparaciones con otras zonas costeras colombianas debido a los pocos estudios realizados al respecto, y a que solo se quiere estudiar el comportamiento de las olas en las costas del departamento del Atlántico-Colombia. 


\section{Ubicación y delimitación de zona objetivo}

La delimitación es importante para la realización de esta investigación, sobre todo la extensión de la costa. En este sentido, se describe la zona inicial del estudio, la cual, como ya se ha dicho, corresponde al departamento del Atlántico, que se encuentra ubicado en la costa norte colombiana, a una

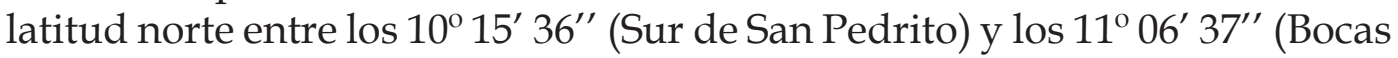
de Ceniza), y a una longitud oeste de Greenwich entre los $74^{\circ} 42^{\prime} 47^{\prime \prime}$ y los $75^{\circ}$ 16' 34" (intersección de Santa Catalina y Arroyo grande). Este departamento limita al oriente con el río Magdalena y el departamento del Magdalena en una longitud de $105 \mathrm{~km}$ desde la desembocadura del río en Bocas de Ceniza hasta la ramificación del canal del Dique a la altura de Suán, Santa Lucía (Atlántico) y Calamar (Bolívar); al norte y noreste con el mar Caribe en una extensión aproximada de $90 \mathrm{~km}$; y al sur, suroccidente y al occidente con el departamento de Bolívar, desde Calamar hasta las Salinas de Galerazamba [3], como aparece en la Fig. 1.

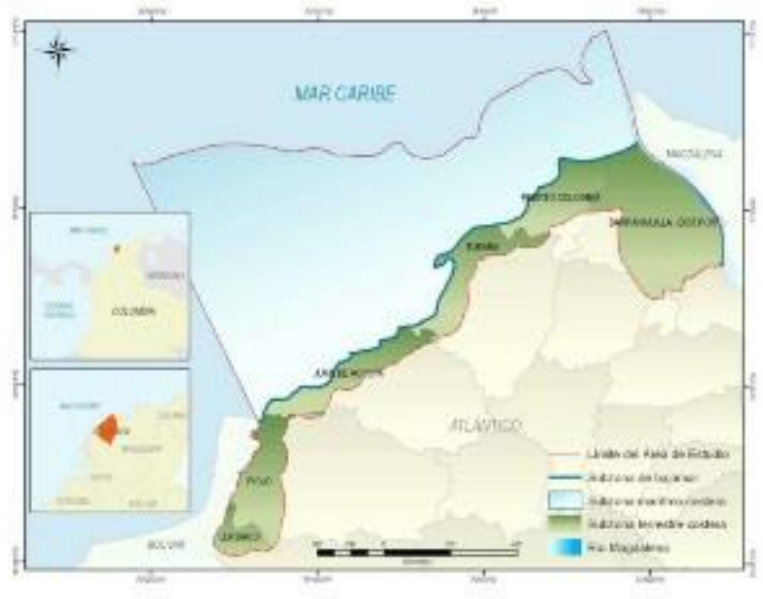

Figura 1.

Ubicación costa

departamento del Atlántico 


\section{Caracterización de las olas en las costas del departamento del atlántico}

Tomando como base la teoría de estudios anteriormente realizados, las olas poseen las características que se muestran en la Fig. 2:

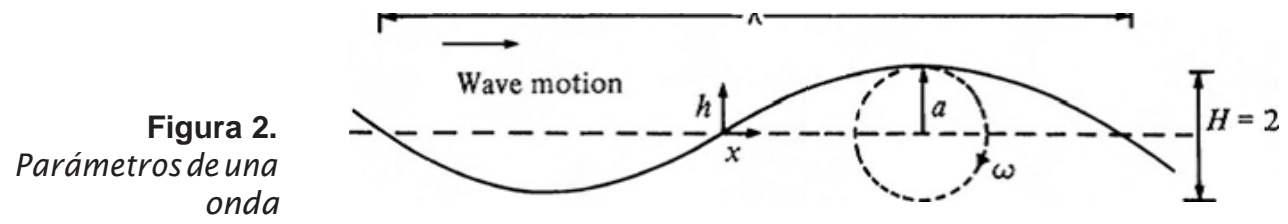

La diferencia de altura entre la cresta y el valle es la altura de la ola $\mathrm{H}$, y la amplitud es la mitad de la altura; la longitud de la onda $\lambda$ es la distancia entre 2 crestas consecutivas, y $\omega$ es la frecuencia angular [4], la cual se muestra en la ecuación (1):

$$
\omega=2 \pi / T
$$

Donde $\Pi$ es una constante matemática aproximadamente igual a 3.1416 y T es el periodo de la onda, entendiendo este último como el tiempo que una onda tarda en recorrer toda su longitud [4].

Otro parámetro de las ondas es su velocidad, también denominada velocidad de fase, que es la velocidad con la que se mueve una onda [4]:

$$
\mathrm{c}=\lambda / \mathrm{T}
$$

La velocidad de fase cambia con relación a la profundidad del mar sobre la 
cual pasan las olas [5], y para aguas profundas (donde la profundidad es mayor a la mitad de la longitud de onda), la velocidad de fase es:

$$
c=g / 2 \pi T
$$

Para aguas poco profundas, donde la profundidad del mar es menor a 1/20 la longitud de la onda (o la longitud de la onda multiplicada por 0.05 ), la velocidad de fase es igual a [5]:

$$
c=\sqrt{g H}
$$

Donde g es la fuerza de gravedad $(9.80665 \mathrm{~m} / \mathrm{s} 2)$ y H es la altura de la ola.

Cuando la profundidad del mar es mayor a la mitad de la longitud de onda y menor que 0.05 por la longitud de la onda, las aguas se consideran intermedias. La fórmula de la velocidad de fase de una ola en estas aguas es [5]:

$\mathrm{c}=\sqrt{ }(\mathrm{g} / \mathrm{k} \tan \mathrm{H}(\mathrm{kH}))$

Donde $\mathrm{H}$ es la altura de la ola, g es la gravedad, y k es el número de onda.

Teniendo en cuenta lo anterior, el área de estudio se caracteriza como aguas de poca profundidad. 


\section{Cuantificación del potencial energetico undimotriz en el departamento del Atlántico}

Los potenciales relativos de los recursos de la energía disponible en las olas de los océanos en el mundo es de alrededor de 2700 GW [6], y el total de energía disponible para usarse es de casi 500 GW. Se estima que la energía undimotriz total que incide en las costas del mundo sobre la superficie del océano es de más o menos 2.11 TW [7]. De acuerdo con varios estudios, en el Océano Atlántico en cercanías a las costas colombianas en el Mar Caribe, el promedio anual de niveles de energía undimotriz oscila entre 10 y $20 \mathrm{~kW} / \mathrm{m}$ de Frente de Ola [1], como se aprecia en la Fig. 3.

Figura 3.

Potenciales de energía Undimotriz en el Mundo. Fuente: Wave Energy paper.

IMechE, 1991 and European Directory of Renewable

Energy (Suppliers and Services) 1991 (c) 2005, Trident Energy Limited.

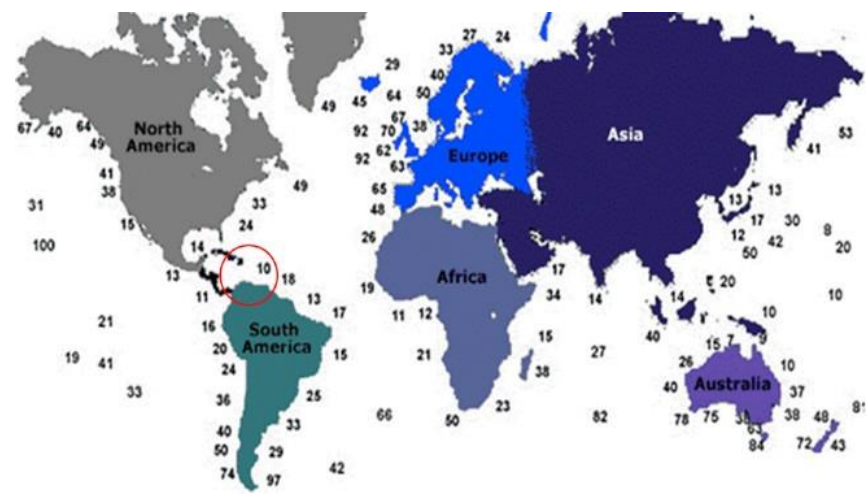

Sin embargo, para la elaboración de este documento se realizaron cálculos basados en datos medidos localmente de las variables que intervienen en la cuantificación del potencial energético. Los resultados serán mostrados posteriormente. 


\section{Ecuaciones Utilizadas}

Teniendo en cuenta todas las consultas bibliográficas realizadas, se seleccionaron las ecuaciones mostradas a continuación, basadas en las aproximaciones con el comportamiento de las olas del mar en la zona delimitada de las costas del departamento del Atlántico, teniendo en cuenta variables como altura significativa y periodo de las mismas. De igual manera, estas formulaciones pueden ser utilizadas para cuantificar el potencial por lo menos en las costas del Mar Caribe colombiano.

En un oleaje idealizado, en el cual la altura y el periodo de las olas es constante, la energía de las olas puede calcularse según la siguiente formula [8]:

$$
\mathrm{P}=\left(\rho g \wedge 2 \mathrm{H}^{\wedge} 2 \mathrm{~T}\right) / 32 \Pi(6)
$$

Donde P: Potencia $(W), \rho$ : Densidad del agua (en este caso agua salada de mar): 1025 kg/m3, g: Gravedad (9.8m/s2), T: Periodo de la ola (s), y H: Altura promedio de la ola $(\mathrm{m})$. Sin embargo, el típico estado del mar está generalmente compuesto por olas que poseen diferentes alturas, periodos, y direcciones. Para un mar irregular, la energía total promedio de las olas se calcula [8]:

$$
\mathrm{P}=\mathrm{kH} \_\mathrm{s}^{\wedge} 2 \text { T_e (7) }
$$

Donde k es una constante que viene dada por la ecuación [9]:

$$
k=\left(\rho g^{\wedge} 2\right) / 64 \Pi
$$

Y donde, a su vez, $\rho$ es la densidad del agua de mar $\left(1025 \mathrm{~kg} / \mathrm{m}^{3}\right)$, g es la gravedad $\left(9.80665 \mathrm{~m} / \mathrm{s}^{2}\right)$ y es una constante matemática cuyo valor aproximado es igual a 3.1416. Ahora ben, como k es una constante y se 
conocen sus variables, basta con hallar su valor y reemplazarlo para que la ecuación de la energía undimotriz quede de la siguiente manera:

$$
\mathrm{P}=490.27 \mathrm{H} \_\mathrm{s}^{\wedge} 2 \mathrm{~T} \_\mathrm{e} \approx 0.49 \mathrm{H} \_\mathrm{s}^{\wedge} 2 \mathrm{~T} \_\mathrm{e}
$$

Donde P es el flujo de energía de la ola expresado en $\mathrm{kW} / \mathrm{m}$, Hs es la altura significativa de la ola y Te es el período en segundos.

La altura significativa Hs se define como la altura promedio del tercio de olas más altas dentro de un grupo de olas considerado. Este valor se aproxima a la altura que un observador en una embarcación reportaría al realizar una inspección visual del estado del mar, ya que tal observador tendería a omitir las olas más pequeñas y menos evidentes. Por otra parte, el periodo de energía Te es el periodo de ola promedio con respecto al espectro de distribución de transporte de energía de las olas [1].

Los mares reales producen olas que poseen alturas, periodos y direcciones aleatorios. Pero en un corto periodo de tiempo, las características de los mares reales permanecen constantes, componiéndose así de un estado del mar [1]. Con el fin de describir estos estados del mar y determinar sus características relevantes para los dispositivos de energía undimotriz, se utilizan parámetros estáticos mencionados antes, los cuales se pueden obtener utilizando dispositivos de medida de las características de las olas como las boyas oceanográficas, también conocidas como boyas de oleaje [10].

La ecuación (9) se utilizó para realizar las primeras estimaciones del posible potencial energético undimotriz Near shore, característicos de las costas del departamento del Atlántico. 


\section{Datos Obtenidos}

Como se ha reiterado, los datos necesarios para aplicar en la ecuación seleccionada se obtuvieron de la base de datos disponible en el $\mathrm{CIOH}$ [2], la cual es una dependencia del DIMAR y estas forman parte del MINDEFENSA. Para conocer las características del oleaje anteriormente descrito, se echó mano de las denominadas boyas oceanográficas, las cuales son utilizadas para registrar las diferentes variables océano-atmosféricas que pueda haber en los mares, como la altura y dirección de las olas, su periodo, la dirección y fuerza del viento, la temperatura tanto del agua como del aire, la presión atmosférica, entre otros.

Estos dispositivos constan de un ordenador que se encarga de recoger la información obtenida de sus medidores en tiempo real, y la envían a través de un sistema de comunicación inalámbrico hacia una estación receptora en tierra para su posterior análisis [10].

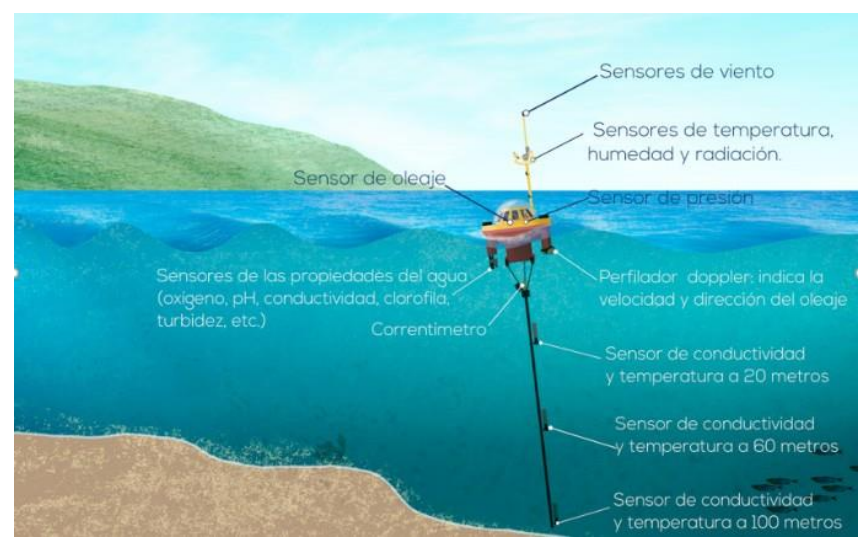

Figura 4.

Esquema de una boya oceanográfica 
Para abastecer de energía los componentes de la boya se hace uso de paneles fotovoltaicos, los cuales se protegen del mar y el viento por medio de una cúpula de vidrio. Por otro lado, la boya se ancla al fondo marino, como se puede ver en la Fig. 4. y en caso de soltarse, posee un sistema de rastreo que permite conocer su ubicación en tiempo real [10].

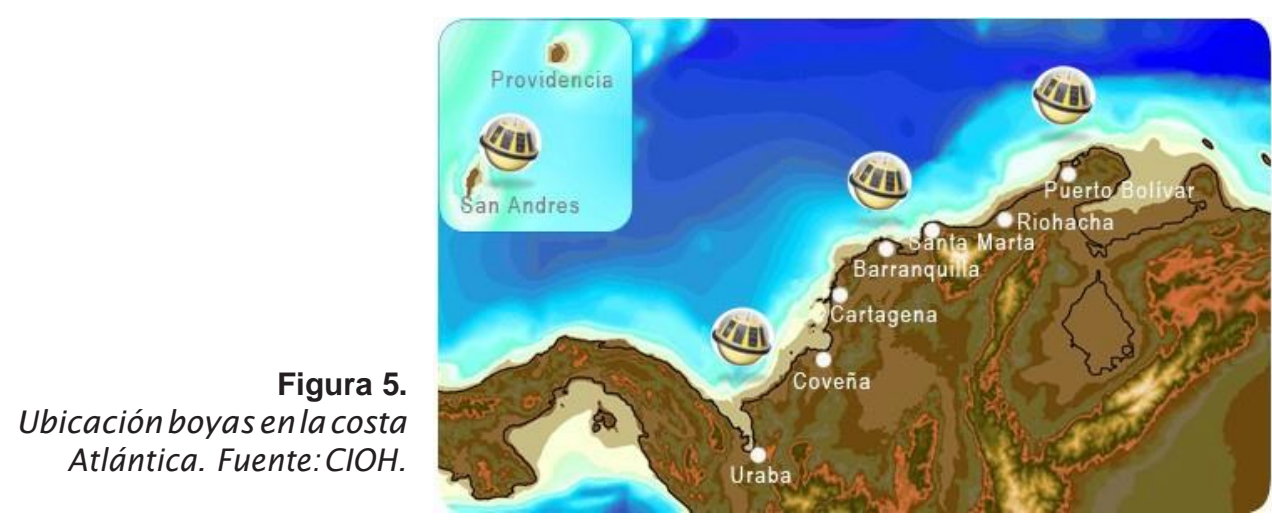

El $\mathrm{CIOH}$ cuenta con 4 boyas oceanográficas ubicadas en el mar Caribe de la siguiente manera: Una en las cercanías a las Islas de San Andrés y Providencia, otra en las Cercanías de Puerto Bolívar en la Guajira, una más en las cercanías de Barranquilla y la última en las cercanías de Coveñas, como se muestra en la Fig. 5.

Para la zona Objetivo se recopilaron datos suministrados por el $\mathrm{CIOH}$ registrados por la boya de oleaje ubicadas en diferentes zonas de la costa del departamento del Atlántico. Como ejemplo, en las Tablas I y II, se muestra información sobre las características del oleaje en diferentes zonas durante algunos días del mes de septiembre del 2016. 


\begin{tabular}{|c|c|c|}
\hline Dia & $\begin{array}{l}\text { Oleaje } \\
(\mathrm{m})\end{array}$ & Periodo $(\mathrm{s})$ \\
\hline 13 & 0.4 & 6.25 \\
\hline 14 & 0.51 & 5.87 \\
\hline 15 & 0.6 & 6.87 \\
\hline 16 & 0.52 & 6.75 \\
\hline 17 & 0.71 & 6.5 \\
\hline 18 & 0.7 & 7 \\
\hline 19 & 0.71 & 7 \\
\hline 20 & 0.55 & 7 \\
\hline
\end{tabular}

Tabla 1.

Oleaje en "el muelle"

(magicseaweed, s.F.)

\begin{tabular}{|c|c|c|}
\hline Dia & $\begin{array}{l}\text { Oleaje } \\
(\mathrm{m})\end{array}$ & Periodo (s) \\
\hline 13 & 0.44 & 6.25 \\
\hline 14 & 0.55 & 5.87 \\
\hline 15 & 0.66 & 6.87 \\
\hline 16 & 0.54 & 6.75 \\
\hline 17 & 0.77 & 6.5 \\
\hline 18 & 0.82 & 7 \\
\hline 19 & 0.74 & 7 \\
\hline 20 & 0.6 & 7 \\
\hline
\end{tabular}

Tabla 2.

Oleaje en "el bolsillo" pradomar (magicseaweed, s.F.)

Se cuenta con datos en otras zonas pertenecientes a las costas del departamento del Atlántico como son: Oleaje en "Punta Roca" (Salgar), Oleaje en "Puerto Velero" y Oleaje en "Charco Grande". Estos datos pueden ser consultados en la base de datos del $\mathrm{CIOH}$, y se han utilizado para realizar esta investigación. 
Para establecer un oleaje promedio en la zona objetivo y obtener información del oleaje más generalizado, además de darle mayor confiabilidad a los cálculos realizados, es necesario hacer un seguimiento al oleaje durante un periodo más amplio que el mostrado anteriormente. De ahí que para la realización de los cálculos realizados en este estudio, se tuvo en cuenta la altura que podían alcanzar las olas en un día dado, entre las fechas del 1 de enero de 2016 y el 31 de enero de 2017 (es decir, durante 13 meses), periodo que se tomó como muestra. Con dicha información se obtuvo la altura significativa del oleaje de cada mes, y se obtuvo mayor precisión en el conocimiento del comportamiento del oleaje. La información utilizada para realizar lo anteriormente descrito se puede encontrar (día por día) en el sitio web del $\mathrm{CIOH}$, así como también, otros datos acerca del mar y el clima en la costa Caribe colombiana. Como ejemplo, en las Fig. 6 y 7 se muestra la altura promedio calculada del oleaje registrado entre los meses de enero y diciembre de 2016:

Figura 6.

Oleaje promedio calculado diario mes de enero 2016.

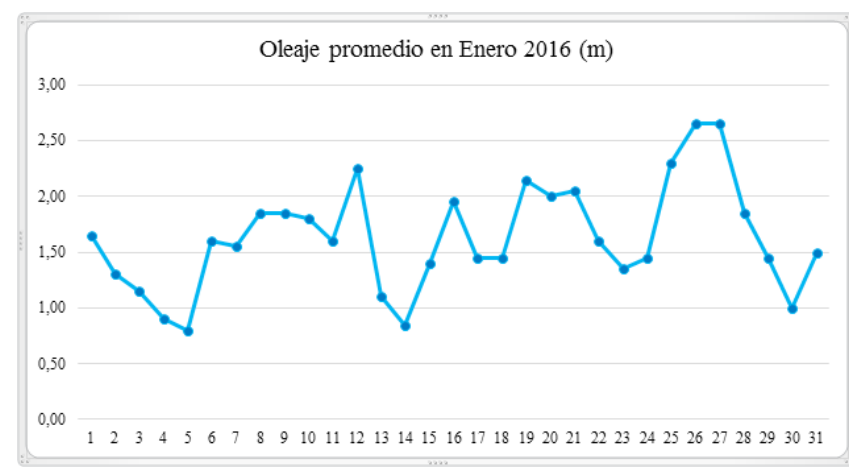

Vol 5 No2 / Julio a Diciembre 2017 


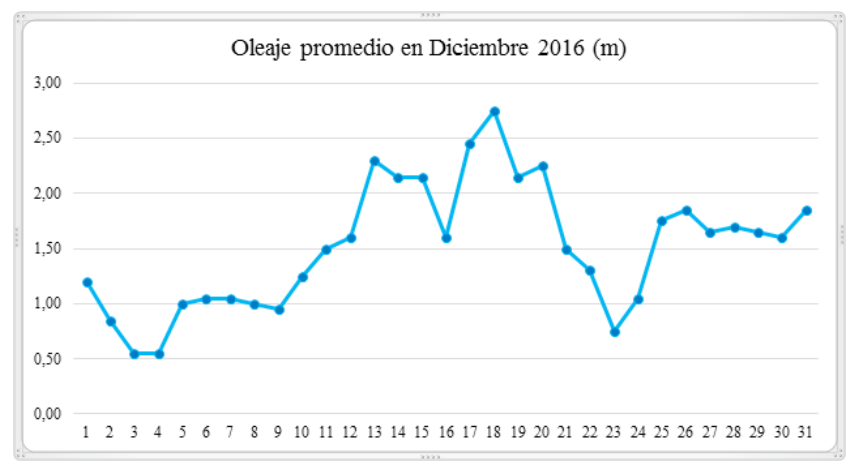

Figura 7.

Oleaje Promedio calculado diario mes de diciembre 2016

\section{Cálculos del potencial energético undímotriz}

Después de obtener la altura promedio a partir de los datos obtenidos, se calculó la altura significativa Hs aplicando el concepto anteriormente descrito. Se tomó el tercio de los datos correspondiente a un mes de los resultados de altura de olas promedio más alto. Es así como en la Fig. 8 se muestra la altura significativa calculada para los 13 meses de registro de datos.

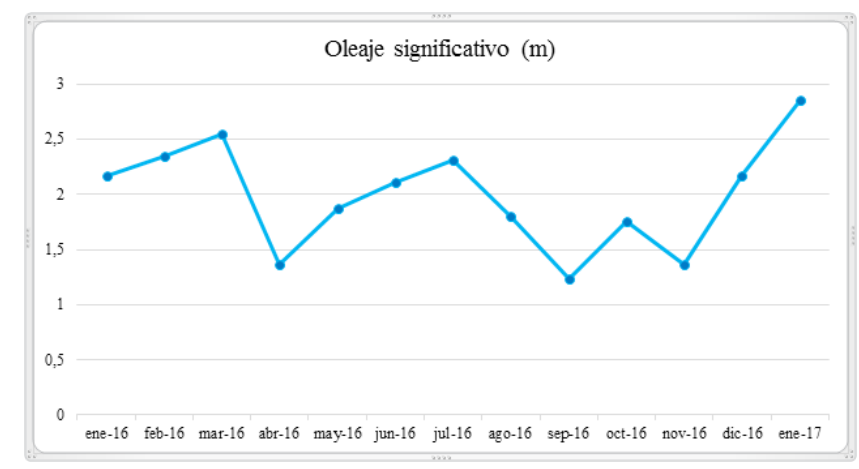

Figura 8.

Altura significativa del oleaje en el Atlántico 
Como se puede observar, el comportamiento del oleaje varía según la época del año y, por ende, el potencial energético también. Usando la ecuación (9) del potencial undimotriz anteriormente descrita, se puede hacer una aproximación del potencial energético undimotriz teórico presente en las costas del departamento del Atlántico. Dicha ecuación (9) tiene en cuenta el periodo de la ola, por lo que, con base en el periodo registrado en distintos puntos de la zona y para efectos prácticos, se utiliza un periodo de 6 segundos. Como ejemplo, para el mes de enero del 2016, la potencia ofrecida $(\mathrm{kW} / \mathrm{m})$ por el oleaje se calculó de la siguiente manera:

$$
\begin{gathered}
\mathrm{P}=0.49 \mathrm{H} \_\mathrm{s}^{\wedge} 2 \mathrm{~T} \\
\mathrm{P}=0.49 \times(2.17 \mathrm{~m})^{\wedge} 2 \times 6 \mathrm{~s} \\
\mathrm{P}=13.84 \mathrm{~kW}
\end{gathered}
$$

De acuerdo con lo inmediatamente anterior, en la Tabla III se muestran los cálculos de potencia de frente de ola realizados teniendo en cuenta la altura significativa Hs de cada mes.

Tabla 3.

Potencia de frente de ola

estimada

\begin{tabular}{|c|c|c|}
\hline Mes & Altura significativa $(\mathrm{m})$ & Potencia $(\mathrm{kW} / \mathrm{m})$ \\
\hline Enero 2016 & 2.17 & 13.84 \\
\hline Febrero 2016 & 2.35 & 16.24 \\
\hline Marzo 2016 & 2.55 & 19.12 \\
\hline Abril 2016 & 1.36 & 5.44 \\
\hline Mayo 2016 & 1.87 & 10.28 \\
\hline Junio 2016 & 2.11 & 13.09 \\
\hline Julio 2016 & 2.31 & 15.69 \\
\hline Agosto 2016 & 1.8 & 9.53 \\
\hline Septiembre 2016 & 1.24 & 4.52 \\
\hline Octubre 2016 & 1.75 & 9.00 \\
\hline Noviembre 2016 & 1.37 & 5.52 \\
\hline Diciembre 2016 & 2.17 & 13.84 \\
\hline Enero 2017 & 2.85 & 23.88 \\
\hline
\end{tabular}


Así, analizando los datos obtenidos en la tabla anterior, los mayores potenciales de energía de las olas se dan entre los meses de enero y marzo, de mayo hasta julio y en el mes de diciembre. Cabe destacar, de todas formas, que en todos los meses se cuenta con un considerable potencial energético. Con los anteriores datos se calcula la potencia total promedio de frente de ola de los meses estimados, lo que da como resultado $12.3 \mathrm{~kW} / \mathrm{m}$. Si tenemos en cuenta la extensión de la costa del Atlántico, que es de $90 \mathrm{~km}$, se halla como resultado un potencial teórico aproximado de 1'107.000 kW o mejor 1107 MW para aplicación de tecnología Near Shore. Para lo anterior, se deben realizar algunos ajustes bajo la premisa de que no sería posible la instalación de tecnología aplicada al aprovechamiento de la energía undimotriz en toda la extensión de la costa, ya que esto dependería de las políticas ambientales locales y de la selectividad de las zonas con mayor potencial. También se debe tener en cuenta la eficiencia de los equipos de conversión de energía undimotriz para ajustar el potencial calculado, que dependerá de la tecnología escogida. Debemos tener en cuenta que este potencial puede aumentar si se aplica tecnología On Shore y Off Shore simultáneamente, las cuales no han sido consideradas aún en este estudio. 


\section{Avances en la verificación del potencial energético}

Con el objetivo de demostrar cuantitativamente el potencial energético contenido en las olas del mar presentadas en el departamento del Atlántico, se está trabajando en el diseño de un sistema de aprovechamiento Near shore, el cual forma parte de las tecnologías denominadas Point absorbers. Los dispositivos Point Absorbers son estructuras flotantes, y tienen componentes que se mueven por la acción de la ola. Estos dispositivos han sido de los más investigados desde el comienzo del estudio y aplicación de la energía undimotriz.

Los "Point Absorbers" utilizan boyas que se mueven junto con las olas, de modo que el movimiento relativo hacia arriba y hacia abajo de las olas que pasan hace que conversores de energía electromecánica o hidráulica generen energía. La forma de la boya no tiene un impacto significativo a la hora de absorber la energía de las olas, debido a que una boya con un tamaño razonable posee un periodo de oscilación mayor que la frecuencia de las olas. Se tiene así que entre más grande sea la boya, más energía puede absorber del mar; sin embargo, si la boya es demasiado grande la ola no podrá pasar sobre ella y no funcionará tampoco el dispositivo "Point Absorber".

De este modo, para hacer buen uso de esta clase de dispositivo, es necesario hacer un diseño que absorba la mayor cantidad de energía que le sea posible teniendo en cuenta el tamaño de la boya, así como también otros factores [11]. En síntesis, si el periodo de oscilación del dispositivo "Point Absorber" coincide con el de las olas que en el inciden, podrá absorber y enviar más energía de las olas hacia el sistema de extracción de energía

El sistema de aprovechamiento que se está diseñando consiste en un dispositivo de este tipo, el cual transforma la energía cinética de las olas del mar en energía mecánica para bombear agua a una determinada altura, convirtiéndola en energía potencial gravitacional que posteriormente, al dejarse caer, puede mover un generador. El esquema del sistema se puede apreciar en la Fig. 9. 


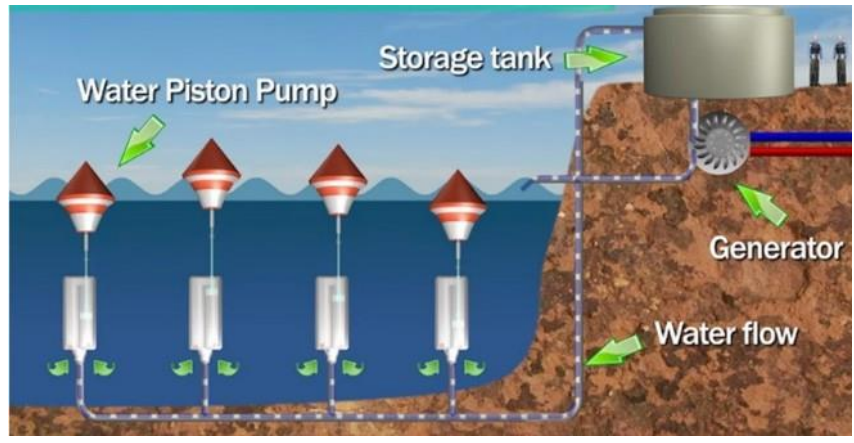

Figura 9.

Esquema del sistema en

etapa de diseño. Fuente:

Blue Energy -- Ocean Power (Piston Pump \& Racks).

Actualmente el proyecto se encuentra en la etapa de diseño de la bomba hidráulica, que bombeará el agua de mar a una determinada altura y se diseñará a escala. Lo anterior permitirá medir el potencial energético real en las costas del departamento del Atlántico, así como sentar las bases para el diseño de un sistema de captación a mayor escala. En la Fig. 10 se muestra el diseño de la bomba.

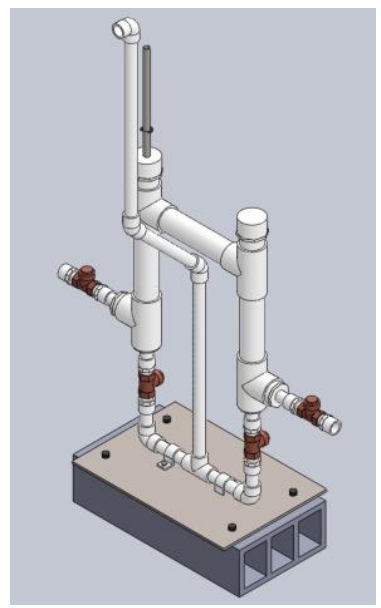

Figura 10.

Diseño bomba hidraulica accionada por boya 
Como puede apreciarse, la bomba está constituida principalmente por 2 cilindros de 7.5 pulgadas (19.05 centímetros) de largo y 1.5 pulgadas (3.81 centímetros) de diámetro, los cuales están unidos en la parte de arriba por una tubería. En uno de los cilindros se encuentran un vástago de 19.68 pulgadas (50 centímetros) de largo y un émbolo. El vástago y el émbolo se encontrarán unidos a una boya calculada de $6 \mathrm{~kg}$ con una geometría definida, que se encarga de bombear el agua hacia las tuberías. A los costados de ambos cilindros hay un agujero en el que se aloja una válvula unidireccional que permite el paso del agua hacia ambos cilindros. La intención de la misma es que, con el movimiento de las olas (ascendente y descendente), la boya haga presión continua suficiente para bombear el agua de mar hasta un tanque de almacenamiento, el cual se encontrará ubicado a una determinada altura; cuando el tanque se llene hasta cierto nivel, se dejará caer el agua a través de un tramo de tuberías para que mueva un generador de corriente alterna, $y$, de esta forma, generar energía eléctrica.

\section{Cálculo de la potencia de la bomba hidráulica}

La potencia que la bomba obtendrá de las olas del mar para bombear el agua hasta el tanque elevado se puede estimar con la siguiente ecuación [12]:

$$
P=Y \times Q \times H \_t
$$

Donde $\mathrm{P}$ es la potencia de la bomba en vatios (W), y es el peso específico del líquido que se va a bombear. El peso específico se halla multiplicando la densidad del líquido, que en este caso es agua de mar (1025 Kg/m3), por la gravedad $(9.80665 \mathrm{~m} / \mathrm{s} 2)$; y esta operación da como resultado que el peso específico del agua de mar es de 10051.81 
$\mathrm{N} / \mathrm{m} 3$, donde $\mathrm{Q}$ es el caudal, el cual posee un valor medido de $0.000145092 \mathrm{~m} 3 / \mathrm{s}$, y H_tes la altura total, a la que se elevará el agua bombeada. 

La altura total de elevación se calcula con la siguiente ecuación [12]:

$$
H \_t=H \_a+H \_f
$$

Donde H_e es la altura de elevación del agua, que para este diseño será de 4 metros $(m)$; y $H_{-}$f son las pérdidas de carga totales que hay en las tuberías en metros $(m)$.

Para hallar las pérdidas totales se tienen en cuenta 2 tipos de pérdidas: las generales, que se encuentran en la tubería de conducción, y las pérdidas específicas, presentes en los accesorios que forman parte de la tubería de conducción.

Hallamos primero las generales, para lo cual se utiliza la ecuación de Darcy-Weisbach [12]:

$$
H \_g=f \times\left(L \_c / D\right) \times\left(v^{\wedge} 2 / 2 g\right)
$$

Donde H_g son las pérdidas de carga en la tubería de conducción debido a la fricción del agua dentro de esta, expresadas en metros; f es el coeficiente de fricción de Darcy- weisbach, un valor adimensional que se calcula teniendo en cuenta el número de Reynolds y la rugosidad relativa (para este diseño, el coeficiente de fricción posee un valor de 0.022); L_c es la longitud de la tubería de conducción en metros (en este diseño se utilizará un tramo de tubería de conducción de 35 metros de largo); D es el diámetro de la tubería de conducción también en metros, el cual será de 0.0127 metros (1.27 centímetros); v es la 
velocidad del agua en la tubería de conducción en metros por segundo $(\mathrm{m} / \mathrm{s})$; y g es la gravedad.

A partir de estos datos, la velocidad, que es el valor que hace falta, se halla de la siguiente forma:

$$
v=Q / A
$$

Donde $Q$ es el caudal en la tubería de conducción, y $A$ es el área de esa misma tubería, el cual es igual a: 


$$
\begin{aligned}
& A=\pi / 4 \times D^{\wedge} 2 \\
& A=\pi / 4 \times(0.0127 m)^{\wedge} 2 \\
& A=0.000126 m^{\wedge} 2
\end{aligned}
$$

Ahora A se reemplaza en la ecuación (14) de la velocidad del agua en

$$
\begin{aligned}
\text { las tuberías: } v & =\left(0.000145092 \mathrm{~m}^{\wedge} 3 / \mathrm{s}\right) / 0.000126 \mathrm{~m}^{\wedge} 2 \\
v & =1.15 \mathrm{~m} / \mathrm{s}
\end{aligned}
$$

Como ya se tiene la velocidad, los valores de la ecuación (13) de Darcyweisbach se pueden reemplazar así:

$$
\begin{gathered}
\mathrm{H} \_\mathrm{g}=0.022 \times((35 \mathrm{~m}) /(0.0127 \mathrm{~m})) \times\left((1.15 \mathrm{~m} / \mathrm{s})^{\wedge} 2 /(2 \times 9.80665\right. \\
\left.\left.\mathrm{m} / \mathrm{s}^{\wedge} 2\right)\right) \mathrm{H} \_\mathrm{g}=0.022 \times 2755.9 \mathrm{~m}^{\wedge} 2 \times 0.0674 \mathrm{~m} \\
\mathrm{H} \_\mathrm{g}=4.08 \mathrm{~m}
\end{gathered}
$$

Las pérdidas en la tubería de conducción debido a la fricción son de 4.08 metros; a estás perdidas generales se le suman las perdidas específicas en los accesorios del tramo de tubería de conducción, para tener un valor más exacto de las pérdidas de carga. Las pérdidas de carga en accesorios se calculan de acuerdo con la siguiente ecuación [12]:

$$
\mathrm{H} \_\mathrm{e}=\mathrm{K} \times \mathrm{v}^{\wedge} 2 / 2 \mathrm{~g}
$$

Donde H_e son las pérdidas especificas en los accesorios de la tubería de conducción, y $\mathrm{K}$ es un coeficiente igual a la suma de las longitudes equivalentes a tubería recta de los accesorios del tramo de tubería de Vol 5 No2 / Julio a Diciembre 2017 
conducción. Dicho tramo tendrá un (1) accesorio T de 90 grados y 2 codos de 45 grados, los coeficientes de $\mathrm{K}$ para la $\mathrm{T}$ y los codos son 1.38 y 0.86 , respectivamente. Sumados estos dos valores dan como resultado un coeficiente $\mathrm{K}$ de 2.24. Ahora se reemplazan los valores en la ecuación

(16) de pérdidas: 


$$
\begin{gathered}
H_{-} e=2.24 \times\left((1.15 \mathrm{~m} / \mathrm{s})^{\wedge} 2\right) /(2 \times 9.80665 \\
\left.\mathrm{m} / \mathrm{s}^{\wedge} 2\right) \mathrm{H} \mathrm{H}_{-}=2.24 \times 0.0674 \mathrm{~m} \\
\mathrm{H} \_\mathrm{e}=0.15 \mathrm{~m}
\end{gathered}
$$

Ya con ambas pérdidas, generales y específicas de los accesorios, se calculan las pérdidas totales en la tubería de conducción para obtener las pérdidas totales $\mathrm{y}$, con estas últimas, hallar la altura total de elevación, reemplazando en la ecuación (12):

$$
\begin{gathered}
H \_t=4 \mathrm{~m}+(4.08 \mathrm{~m}+0.15 \mathrm{~m}) \\
\mathrm{H} \_\mathrm{t}=4 \mathrm{~m}+4.23 \mathrm{~m} \\
\mathrm{H} \_\mathrm{t}=8.23 \mathrm{~m}
\end{gathered}
$$

Conociendo todas las variables necesarias para calcular la potencia de la bomba hidráulica, solo queda reemplazarlos en la ecuación (11) y resolverla:

$$
\begin{gathered}
\mathrm{P}=10051.81 \mathrm{~N} / \mathrm{m}^{\wedge} 3 \times 0.000145092 \\
\mathrm{~m}^{\wedge} 3 / \mathrm{s} \times 8.23 \mathrm{~m} \mathrm{P}=12 \mathrm{~W}
\end{gathered}
$$

\section{Cálculo del peso de la boya}

Para calcular este valor se utiliza la siguiente ecuación:

$$
\text { Peso_boya }=\left(\left(A \_i \times D \_c\right) \times \rho\right)+\left(\left(A \_c \times L \_c\right) \times \rho\right)
$$

Donde:

A_i = área de la tubería de impulsión, donde se bombea el agua (0.00114 m2) 
D_c = Distancia de la camisa de la bomba, desde el émbolo (en su

posición más alta) hasta el fondo de camisa, esta distancia es de 22.5

$\mathrm{cm}(0.225 \mathrm{~m})$

A_c $=$ es el área del tramo de tubería de conducción

$(0.000126 \mathrm{~m} 2) \mathrm{L} \_\mathrm{C}=$ es la longitud del tramo de tubería

de conducción (35 m)

$\rho=$ es la densidad del agua de mar 
Al multiplicar A_ixD_c y $A \_c \times L \_c$, se obtienen los volúmenes de la camisa de la bomba y el del tramo de tubería de conducción, respectivamente. Sabiendo esto, la ecuación puede simplificarse y quedará como:

$$
\text { Peso_boya }=\left(\left(V_{-} i\right) \times \rho\right)+\left(\left(V_{-} c\right) \times \rho\right)(18)
$$

En la que $V_{-} i$ es el volumen de la camisa $(0.000256 \mathrm{~m} 3)$, y $V_{-} c$ es el volumen del tramo de tubería de conducción $(0.00441 \mathrm{~m} 3)$ :

Peso_boya $=\left(0.000256 \mathrm{~m}^{\wedge} 3 \times 1025 \mathrm{~kg} / \mathrm{m}^{\wedge} 3\right)+\left(0.00441 \mathrm{~m}^{\wedge} 3 \times 1025 \mathrm{~kg} / \mathrm{m}^{\wedge} 3\right.$

) Peso_boya $=(0.26 \mathrm{Kg})+(4.52 \mathrm{~kg})$

Peso_boya $=4.78 \mathrm{~kg}$

De acuerdo con el resultado, la boya debe tener un peso mínimo de $4.78 \mathrm{~kg}$, aunque preferiblemente su peso debe ser mayor al resultado de la fórmula para garantizar un funcionamiento óptimo de la bomba hidráulica. Por esta razón y por efectos prácticos, se ha decidido que la boya para el dispositivo será de 6,6 kg de peso, por encontrar en el mercado pesas calibradas de $2,2 \mathrm{~kg}$. 



\section{Conclusiones}

Es evidente que existe un potencial energético undimotriz en las costas del Caribe colombiano, y con este trabajo se está dando el primer paso para ser identificado, cuantificado y utilizado. Apoyándonos en los resultados obtenidos en las costas del departamento del Atlántico, el potencial undimotriz teórico estimado resulta interesante para la aplicación de tecnologías que aprovechen este tipo de energía. Por otra parte, si bien los datos nos orientan hacia la aplicación de tecnologías Near Shore, estos nos pueden servir como referencia para las aplicaciones On shore y off shore aumentando el potencial estimado. Los cálculos realizados para la bomba hidráulica a escala que se propone arrojan unos resultados que hacen factible la obtención de energía eléctrica en las olas de las costas del Atlántico teniendo en cuenta las características particulares del oleaje. 


\section{Referencias Bibliográficas}

1. J. Brooke, Wave Energy Conversion, Primera ed., vol. VI, Elsevier Science, 2003.

$2 \mathrm{CIOH}$, "INICIO || CIOH.ORG - Oceanografica operacional", [En línea]. Available: https://www.cioh.org.co/meteorologia/index.php.

3. Wikipedia, «Atlántico (Colombia),» [En línea]. Available: https:// es.wikipedia.org/wiki/Atl\%C3\%A1ntico_(Colombia). [Último acceso: 20 Abril 2017].

4. J. J. Muñoz Pérez, Ondas regulares y su interpretación a la ingenieria de costas, Cádiz: Servicio de publicaciones de la universidad de Cádiz, 2011. 5. R. G. Dean y R. A. Dalrymple, Water Wave Mechanics for Engineers and Scientists.

6. M. E. McCormick y C. Ertekin, "Renewable Sea Power", Mechanical Engineering, Mayo 2009.

7. K. Gunn y C. Stock-Williams, Quantifying the global wave power resource, 2012.

8. L. Duckers, Wave Energy.

9. A. Kies, B. Schyska y L. Von Bremen, Energy Reports 2.

10. MEDCLIC, "Infraestructuras fijas", [En línea]. Available: http:// www.medclic.es/es/instrumentos/infraestructuras-fijas/. [Último acceso: 14 Febrero 2017].

11. J. Engström, "Hydrodynamic Modelling for a Point Absorbing Wave Energy Converter" 2011. [En línea]. Available: http://uu.diva-portal.org/ smash $/$ record.jsf? pid=diva2\%3A450881\&dswid=2970. [Último acceso: 17 Enero 2017].

12 UGR, "Calculo de bombas y tuberías", [En línea]. Available: http:// www.ugr.es/ aulavirtualpfciq/Bbombasytuberias.html. [Último acceso: 18 Mayo 2017]. 


\section{Bibliografía de consulta}

Fagro, "Hidráulica en tuberías a presión", 2015. [En línea]. Available: http:/ / www.fagro.edu.uy/hidrologia/riego/HIDRAULICA\%202015.pdf. [Último acceso: 18 Mayo 2017].

A. Khaligh y O. C. Onar, Ocean Wave Energy Harvesting, 2010.

A. F. d. O. Falcão, "Wave Energy Utilization”, A Review of the Technologies, 2009.

Agencia Estatal de Meteorología - AEMET, Untitled, [En línea]. Available: http:/ / www.aemet.es/documentos/es/conocermas/maritima/escalas_ de_viento_y_oleaje.pdf. [Último acceso: 14 Febrero 2017].

Atmocean, Technology, [En línea]. Available: https://atmocean.com/ technology/. [Último acceso: 23 Noviembre 2016].

Boreau of Ocean Energy Management (BOEM), Ocean Wave Energy, [En línea]. Available: https://www.boem.gov/Ocean-Wave-Energy/. [Último acceso: 22 Noviembre 2016].

Euskadi, «La planta de las olas de Mutriku alcanza un hito de producción mediante la energía renovable de las olas al generar más de 1GWh,» 18 Julio 2016. [En línea]. Available: http://www.euskadi.eus/gobiernovasco/-/noticia/2016/la-planta-de-las-olas-de-mutriku-alcanza-un-hitode-produccion-mediante-la-energia-renovable-de-las-olas-al-generar-masde-1gwh/. [Último acceso: 23 Noviembre 2016].

European Marine Energy Centre (EMEC), About Us, [En línea]. Available: http:/ / www.emec.org.uk/about-us/. [Ultimo acceso: 16 Enero 2017].

J. Twidell y T. Weir, Renewable Energy Resources, 2006.

M. F. Tovar Rojas y L. F. Yamá Mosquera, Bogotá D.C., 2009.

C.-k. Kim, J. E. Toft, M. Papenfus, G. Verutes, A. D. Guerry, M. H. Ruckelshaus, et al, "Catching the Right Wave: Evaluating Wave Energy Resources and Potential Compatibility with Existing Marine and Coastal Uses", vol. VII, 2012.

Office of Energy Efficiency \& Renewable Energy (EERE), Prototype Testing Could Help Prove a Promising Energy Source, 8 Junio 2015. [En línea]. Available: http://energy.gov/eere/articles/prototype-testing-could-helpprove-promising-energy-source. [Último acceso: 23 Noviembre 2016]. 


\section{Como Citar}

J. Pérez, y W. Becerra,'Cuantificación del potencial energético undimotriz en las costas del Caribe colombiano", Investigación e Innovación en Ingenierias, vol. 5, n . 2, pp. 110-137., 2017 